\title{
Chemopreventive Synergism between Green Tea Extract and Curcumin in Patients with Potentially Malignant Oral Disorders: A Double-blind, Randomized Preliminary Study
}

\author{
Mellekatte C Neetha ${ }^{1}$, Mamatha G Panchaksharappa ${ }^{2}$, Shashikala Pattabhiramasastry ${ }^{3}$, Nandish V Shivaprasad ${ }^{4}$, \\ Usha G Venkatesh ${ }^{5}$
}

\begin{abstract}
Aim: The aim of the study was to assess the synergistic effect of green tea extract and curcumin in patients with oral potentially malignant disorders (OPMDs) and to ascertain the mechanism of action of these chemopreventive agents through assessment of suitable biomarkers.

Materials and methods: Subjects with OPMDs $(n=60)$ were randomized to receive green tea extract [topical + systemic $(800 \mathrm{mg} / \mathrm{day})]$ or curcumin [topical + Systemic ( $950 \mathrm{mg} /$ day)] or a combination therapy with 20 patients in each group for 3 months. Biomarkers (Ki67, cyclin D1, and p53) were evaluated in baseline and 12-week biopsies.

Results: The clinical response rate observed in OPMDs was higher in the combination group $(n=13 ; 65 \%)$ as compared to the curcumin ( $n=11$; $55 \%)$ and the green tea extract group $(n=7 ; 35 \%)$ and was statistically highly significant. Treatment medications also improved histological grades, although not statistically significant. All the study drugs were well tolerated by patients and did not raise any safety concerns. There was statistically significant $(p<0.01)$ downregulation of p53, Ki67, and cyclin D1 expression at 3 months as compared to baseline in the combination group.

Conclusion: Treatment of OPMDs with curcumin and green tea extract combination demonstrated a significant clinical response supported by downregulation of molecular biomarkers in the short-term (12 weeks). The present results warrant a long-term clinical testing of green tea and curcumin combination for oral cancer prevention.

Clinical significance: Chemoprevention is a promising treatment strategy to reverse, stabilize, or arrest progression of these OPMDs. Use of natural dietary agents like green tea and curcumin, which are readily available, have low toxicity, and more importantly demonstrate a synergistic effect, is an attractive alternative in the chemoprevention of oral cancer. The assessment of biomarkers has helped us to understand the mechanism of action of these chemopreventive agents.

Keywords: Chemoprevention, Curcumin, Green tea extract, Oral potentially malignant disorders, Randomized preliminary study, Synergistic effect. The Journal of Contemporary Dental Practice (2020): 10.5005/jp-journals-10024-2823
\end{abstract}

\section{INTRODUCTION}

Oral cancer often stems from potentially malignant disorders (PMDs) like leukoplakia, oral submucous fibrosis (OSMF), and erosive lichen planus, which carry a $2-3 \%$ overall possibility of transforming into malignancy. This risk surges by fivefold within 8 years for dysplastic or high-risk PMDs such as erythroleukoplakia, erythroplakia, and proliferative verrucous leukoplakia. ${ }^{1}$

Chemoprevention is a revolutionary method to reverse, stabilize, or halt progression of these PMDs and an effective chemopreventive agent must be efficient and harmless for extended use. Most widely studied chemopreventive agents were retinoids and their use was restrained because of their toxicity. ${ }^{2,3}$ Green tea and curcumin are natural dietetics and both have been studied for their chemopreventive capability because of their ready availability, low toxicity, and potential ability to inhibit carcinogenesis, decrease risk of cancers, and most importantly prevent second primary cancers due to the process of field cancerization. ${ }^{4,5}$

In several animal models, polyphenols, especially epigallacatechin-3-gallate (EGCG) found in green tea, have demonstrated anticarcinogenic activity by showing downregulation of cell growth or proliferation markers like cyclin D1, Ki 67, arrest of cells in the G0-G1 phase, stabilize p53, and regulated apoptotic cell death. ${ }^{6,7}$ Curcumin, a key polyphenol extracted from the Indian yellow spice turmeric, has also demonstrated to have

\begin{abstract}
1,2Department of Oral Medicine and Radiology, College of Dental Sciences, Davangere, Karnataka, India

${ }^{3,4}$ Department of Pathology, SS Institute of Medical Sciences and Research Centre, Davangere, Karnataka, India

${ }^{5}$ Department of Preventive and Public Health Dentistry, Bapuji Dental College and Hospital, Davangere, Karnataka, India
\end{abstract}

Corresponding Author: Mellekatte C Neetha, Department of Oral Medicine and Radiology, College of Dental Sciences, Davangere, Karnataka, India, Phone: +919900207778, e-mail: neethamc@gmail. com

How to cite this article: Neetha MC, Panchaksharappa MG, Pattabhiramasastry $\mathrm{S}$, et al. Chemopreventive Synergism between Green Tea Extract and Curcumin in Patients with Potentially Malignant Oral Disorders: A Double-blind, Randomized Preliminary Study. J Contemp Dent Pract 2020;21(5):521-531.

Source of support:The study was funded by Rajiv Gandhi University of Health Sciences, Bangalore, Karnataka, India

Conflict of interest: None

anticancer characteristics like disruption of expression of several proto-oncogenes, targeting numerous molecular endpoints, as well as integration of mutagens to cellular DNA. ${ }^{5}$ Curcumin treatment reduces mutant p53, cyclin D1, and expression of proliferative marker Ki67 when investigated both on cell lines and 
the animal model. ${ }^{8-10}$ The sole means to establish the working of any chemopreventive agent toward a response and opposition at the molecular level is by assessing appropriate biomarkers.

An in vitro study has shown EGCG from green tea arrested cells in the $G_{0}-G_{1}$ phase, while curcumin invoked an $S / G_{2} M$ block; ${ }^{11}$ combination of EGCG and curcumin has also shown to suppress keratinocyte proliferation and survival, ${ }^{12}$ and EGCG potentiates the effect of curcumin in inducing growth inhibition and apoptosis of resistant breast cancer cells. ${ }^{13}$ Based on this differential mechanism of growth hindrance, we postulate that combination treatment would show more efficacy than a single agent.

So, the present study has been designed to examine the chemopreventive potential of green tea extract and curcumin on PMDs, when used as single agents or as combination, and further validating the results of the preliminary study by assessing suitable molecular biomarkers like p53, cyclin D1, and Ki 67.

\section{Materials and Methods}

\section{Study Design and Patient Selection}

Green tea extract and curcumin chemoprevention in oral potentially malignant disorders (OPMDs) was a randomized, double-blind preliminary study to test the efficacy of chemopreventive agents when used singly or as combination, which was conducted at Department of Oral Medicine and Radiology, College of Dental Sciences, Davangere. This study was funded by Rajiv Gandhi University of Health Sciences, Bengaluru, Karnataka. The study procedure was accepted by the College of Dental Sciences Institutional Review Board and was carried out in compliance with the requirements of the Declaration of Helsinki and Good Clinical Practice Guidelines.

The principle inclusion criterion was existence of OPMDs, which were histologically confirmed and bidimensionally assessable. Additional inclusion criteria include patients with adequate hematologic liver, renal, and cardiac functions; a written informed consent for participation; willingness to quit the habit of tobacco smoking/chewing and alcohol use; and readiness to visit during follow-ups. The exclusion criteria were as follows: known hypersensitivity to oral green tea extract, curcumin and its analogs, prior use of chemopreventive agents within 30 days, history of prior malignancy, pregnancy or lactation, and patients unable to take oral medications.

On enrolment, all subjects were given an account of the study protocol and provided an informed consent. A detailed medical history (including routine use of alcohol, tobacco, use of simultaneous drugs and their adverse effects) was taken and were subjected for general physical check, two-dimensional measurement, and a biopsy of OPMD. Intensity of any associated symptoms like pain, burning, or substantial discomfort was measured using the visual analog scale (VAS) of $0-10$. The participants were counseled and guided to quit the habit completely.

The sample size was planned based on parallel reported studies on OPMDs. ${ }^{1,14}$ The sample size was estimated using $\mathrm{G}$ power 3.1.9.4. We assumed a priori $20 \%$ of the participants would be lost to follow-up by 3 months. $a$ and power of the study were fixed at 0.05 and $80 \%$, respectively. This suggested a minimum sample size of 20 per group. Considering reasons like denial to participate, loss to follow-up, or others, the sample size was increased to 30 in each group.

To balance prognostic factors in each arm, the Pocock-Simon dynamic allocation scheme was used for randomization. ${ }^{15}$ The prognostic factors were consumption of tobacco (never, former, current) and alcohol (never, occasional, current). Suitable patients were randomized to one of the three treatment arms equally in a double-blinded manner wherein both investigator and patients were not shown the treatment assignments except the study supervisor.

The three treatment arms were the following:

- Green tea extract group (topical + systemic)

- Curcumin group (topical + systemic)

- Combination group

Based on the previous studies, the dose for green tea extract used was $400 \mathrm{mg}$ BID and curcumin $475 \mathrm{mg}$ BID. ${ }^{1,16}$ Both the capsules were purchased from Zenith Nutrition, Bengaluru. Topical preparation of curcumin was purchased from Abbott Healthcare Pvt. Ltd. (curenext oral gel $50 \mathrm{~g}$ ). Topical preparation of green tea extract in the form of gel was prepared using the green tea extract capsules from Bapuji College of Pharmacy. All patients received treatment for duration of 3 months. During the study period, both safety and clinical efficacy assessment was done every 4 weeks. One-month quantity-of-study medications were given to patients every 4 weeks and were informed to note down abidance in a diary and to handover any remaining medicines if left out. Study supervisors checked the dairies and left-out capsules were counted every 4 weeks throughout the active treatment period. A patient was contemplated compliant if it was found that the $80 \%$ of the prescribed medications were consumed during the study. Toxicity before initiating each 4-week cycle was assessed conferring to the National Cancer Institute Common Terminology Criteria for Adverse Events (CTCAE) Version 4.0. ${ }^{17}$ Any grade II, III, or IV toxicity, study medication was withdrawn. Subjects were dropped from study if the consent was withdrawn, wished discontinuation due to any concurrent ailment, were pregnant or unsuccessful to use appropriate birth control, were reluctant to conform to the study procedure and follow-up, or died.

The histologic and biomarker assessment was done at baseline and after 3 months of treatment. Tissue biopsies (at baseline and 3 months) were subjected to immunohistochemistry (IHC) at Department of Pathology, SS Institute of Medical Sciences and Research Center, for the assessment of the biomarkers mentioned here: cell growth/proliferation markers-Ki-67, cyclin D1; and apoptosis marker-p53. All biopsy slides were reviewed for suitability for analysis.

\section{Biomarker Assay}

Immunohistochemistry - tissue biopsies (at baseline and 3 months) were evaluated by IHC in the most histologically advanced areas of basal and suprabasal layers for nuclear staining of p53, Ki-67, and cyclin D1. After processing the biopsies, IHC was carried out as per regular practice using appropriate antibodies. The $4 \mu \mathrm{M}$ thin unstained slides were obtained from formalin-fixed and paraffin-embedded tissue sections for IHC analyzes. After deparaffinization, the slides were hydrated, and microwaved for 10 minutes with $10 \mathrm{mmol} / \mathrm{L}$ of Tris EDTA buffer for antigen retrieval, and bathed in Tris buffer. The slides were incubated with the primary and then secondary antibodies after treating with $3 \% \mathrm{H}_{2} \mathrm{O}_{2}$ in methanol to occlude nonspecific binding. Specifically diluted mouse monoclonal primary antibodies against p53-BP-5312 (Pathnsitu Biotechnologies, India) and Ki67-GM001 (Pathnsitu Biotechnologies) and rabbit monoclonal primary antibody against cyclin D1-EP12 (pathnsitu biotechnologies) were used for incubation of the sections. Successively, the sections were incubated at room 
temperature with a secondary antibody (polyexcel HRP/DAB detection system, Pathnsitu Biotechnologies), and then they were processed with $0.5 \mathrm{mg} / \mathrm{mL}$ DAB solution having $0.001 \% \mathrm{H}_{2} \mathrm{O}_{2}$ to visualize brown-colored end products, and counterstained with hematoxylin.

Two pathologists ( $\mathrm{S}$ K and N, who were both blinded to the clinical and histological parameters) assessed IHC staining individually using light microscopy (200x magnification). The intensity of staining (range $0-3$ ) and percentage of positive cells (range 1-100\%) were estimated as described in previous publications. ${ }^{1}$ The intensity of staining is recorded as follows: 0 , no noticeable staining; 1 , weak staining compared with the stromal elements; 2 , intermediate staining; 3 , strong if dark brown staining was evident. A nuclear IHC staining for cyclin D1, Ki-67, and p53 in the basal and suprabasal epithelium was assessed. The labeling index (LI) was determined by dividing the sum of positive cells by the total number of cells counted in each slide and multiplying by 100. One thousand cells were counted in each slide.

\section{Study Endpoints}

The primary endpoints were clinical, histologic response and effect of medications on the expression of biomarkers in OPMDs at 3 months among three different study groups. The secondary endpoint was to appraise the safety of study drugs.

The clinical responses were determined by comparing the size of the lesion at baseline and at 3 months: complete response $(C R)$, waning of an entire lesion; partial response (PR), $50 \%$ or more reduction in the sum of products of diameters of all the lesions; progressive disease (PD), more than $25 \%$ increase in size of lesion; and stable disease (SD) were considered if the response did not meet criteria for CR, PR, or PD. The histologic response criteria were as follows: $\mathrm{CR}$, total reversal of dysplasia/hyperplasia to normal epithelium; PR, downgrade in the degree of dysplasia; $\mathrm{SD}$, no change in the degree of dysplasia; and histologic PD, any advancement in the severity grade. ${ }^{1}$

\section{Statistical Methods Employed for Analysis of Data}

Procured data were assembled on a MS Office Excel Sheet (v 2010, Microsoft Redmond Campus, Redmond, Washington, United States) and statistical analysis was done using the Statistical package for Social Sciences (SPSS v 21.0, IBM).

Descriptive statistics like frequencies and percentage for categorical data and mean and SD for numerical data have been depicted. Regularity of numerical data was assessed using the Shapiro-Wilk test and as the data did not follow a normal curve, nonparametric tests have been used for comparisons. Intergroup comparison (more than two groups) was done using Kruskal-Wallis ANOVA and subsequently pairwise comparison was made using the Mann-Whitney U test. Intragroup comparison was done using the Wilcoxon Signed rank test (up to two observations). Comparison of frequencies of categories of variables with groups was done using the Chi-square test. Age has been compared using the one-way ANOVA test. Concordance between histologic and clinical response was evaluated within each treatment arm using the McNemarBowker's test. Risk profiles like tobacco and alcohol consumption were compared with clinical and histologic response rates and also with baseline biomarker data using the Wilcoxon rank-sum test or the Kruskal-Wallis test. The difference in biomarker variation was linked between responders and nonresponders and among treatment groups using the Wilcoxon rank-sum test. All tests were two-sided and were considered statistically significant if $p \leq 0.05$.

\section{Results}

\section{Patient and Treatment Characteristics}

Of the 220 subjects screened between March 2016 and May 2018, 90 fulfilled all the inclusion and exclusion criteria and 30 each were allocated randomly to green tea arm, curcumin arm, and combination arm. A total of 23 patients were lost to follow-up and 7 patients discontinued intervention for other reasons like noncompliance or withdrawal of consent due to personal reasons. The final sample consisted of 60 subjects with OPMDs. Steps involved in the present study are comprehensively represented in the form of a flowchart (Flowchart 1).

The demographic, risk profile, clinical, and histologic characteristics of the patients of all the three groups are seen in Table 1.

Flowchart 1: CONSORT flow chart showing patient disposition

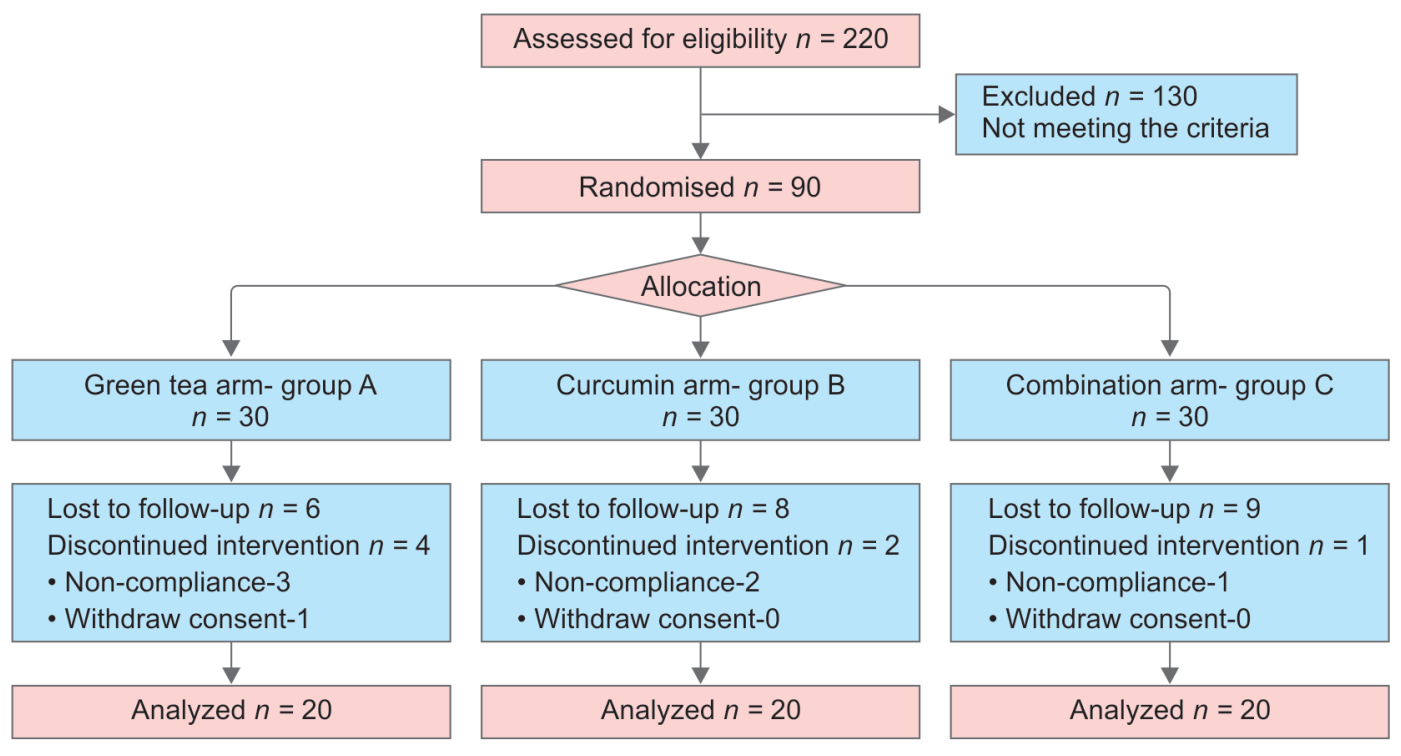


Table 1: Demographic details of participants

\begin{tabular}{|c|c|c|c|c|}
\hline Demographic variables & Group I $(n=20)$ & Group II $(n=20)$ & Group III $(n=20)$ & Total $(n=60)$ \\
\hline Median age (range) & $40.5(16-82)$ & $42(19-65)$ & $54.5(22-71)$ & $45.5(16-82)$ \\
\hline \multicolumn{5}{|l|}{ Gender, $n(\%)$} \\
\hline Male & $15(75)$ & $17(85)$ & $13(65)$ & $45(75)$ \\
\hline Female & $5(25)$ & $3(15)$ & $7(35)$ & $15(25)$ \\
\hline \multicolumn{5}{|l|}{ Smoking status, $n$ (\%) } \\
\hline Never & $11(55)$ & $11(55)$ & $11(55)$ & $33(55)$ \\
\hline Former & $1(5)$ & $1(5)$ & $2(10)$ & $4(6.6)$ \\
\hline $5-10$ years & $1(5)$ & $2(10)$ & $2(10)$ & $5(8.4)$ \\
\hline$>10$ years & $7(35)$ & $6(30)$ & $5(25)$ & $18(30)$ \\
\hline \multicolumn{5}{|c|}{ Smokeless tobacco status, $n(\%)$} \\
\hline Never & $6(30)$ & $4(20)$ & $5(25)$ & $15(25)$ \\
\hline Former & $2(10)$ & $1(5)$ & $3(15)$ & $6(10)$ \\
\hline Current $<5$ years & $0(0)$ & $2(10)$ & $1(5)$ & $3(5)$ \\
\hline $5-10$ years & $5(25)$ & $5(25)$ & $6(30)$ & $16(26.6)$ \\
\hline$>10$ years & $7(35)$ & $8(40)$ & $5(25)$ & $20(33.4)$ \\
\hline \multicolumn{5}{|l|}{ Alcohol status, $n$ (\%) } \\
\hline Never & $13(65)$ & $12(60)$ & $13(65)$ & $38(63.4)$ \\
\hline Occasional & $4(20)$ & $6(30)$ & $5(25)$ & $15(25)$ \\
\hline $5-10$ years & $3(15)$ & $2(10)$ & $2(10)$ & 7 (11.6) \\
\hline \multicolumn{5}{|l|}{ Baseline histology, $n$ (\%) } \\
\hline Hyperplasia & $9(45)$ & $8(40)$ & $8(40)$ & $25(41.6)$ \\
\hline Mild dysplasia & $2(10)$ & $6(30)$ & $6(30)$ & $14(23.3)$ \\
\hline Moderate dysplasia & $8(40)$ & $5(25)$ & $6(30)$ & 19 (31.6) \\
\hline Severe dysplasia & $1(5)$ & $1(5)$ & $0(0)$ & $2(3.3)$ \\
\hline \multicolumn{5}{|c|}{ Baseline clinical staging, ${ }^{44-46} n$} \\
\hline Phase 2 leukoplakia & 13 & 7 & 3 & 23 \\
\hline Phase 4 leukoplakia & 0 & 4 & 6 & 10 \\
\hline Grade II OSMF & 0 & 0 & 1 & 1 \\
\hline Grade III OSMF & 0 & 0 & 1 & 1 \\
\hline Score 1 lichen planus & 0 & 1 & 0 & 1 \\
\hline Score 2 lichen planus & 0 & 3 & 0 & 3 \\
\hline Score 3 lichen planus & 5 & 3 & 3 & 11 \\
\hline Score 5 lichen planus & 2 & 2 & 6 & 10 \\
\hline
\end{tabular}

OSMF, oral submucous fibrosis

The distribution of smokers $(p=0.786)$, tobacco chewers $(p=0.836)$, alcohol users $(p=0.947)$, age $(p=0.205)$, sex $(p=0.344)$, and baseline histology ( $p=0.663)$ was comparable among all the three groups. Baseline clinical staging showed a higher frequency of phase 2 leukoplakia in group I and also more number of phase 4 leukoplakia and score 5 lichen planus in group III $(p=0.014)$.

\section{Efficacy}

All the three groups showed reduction in the intensity of associated symptoms at the end of 3 months as depicted by decrease in VAS scores in Figure 1.

Clinical response rates are represented in Figure 2A. There was a significant difference seen for the frequencies between the groups ( $p=0.023$ ) with higher frequency of clinical response in the combined group. The clinical response ( $C R+P R)$ was observed in 13 subjects (65\%) in the combination arm, 11 subjects $(55 \%)$ in the curcumin arm, and 7 (35\%) subjects in the green tea arm. A total of 7 (35\%) subjects in the combination arm, 9 (45\%) subjects in the curcumin arm, and $13(65 \%)$ subjects in the green tea arm were nonresponders (SD + PD). Statistically significant difference in clinical response rates was noted in the combination (curcumin + green tea) arm and the curcumin arm as compared to the green tea arm. Statistically nonsignificant difference in histologic response rates was observed between all the treatment groups $(p=0.670)$. The histologic response $(C R+P R)$ was observed in three subjects (15\%) in the combination arm, seven subjects (35\%) in the curcumin arm, and six (30\%) subjects in the green tea arm (Fig. 2B). Among 31 clinical responders, 18 had hyperplasia, 6 had mild dysplasia, and 7 patients had moderate dysplasia in their baseline biopsy. In 16 patients with a histologic response, 3 had hyperplasia, 5 had mild dysplasia, and 8 had moderate dysplasia. On comparison of 3-month clinical or histologic response with risk profile, no statistically significant correlation was found; however, we found a higher clinical and histologic response rate in never tobacco smokers and never drinkers. When we assessed concordance among histologic and clinical response within each treatment group (McNemars-Bowker's test), there was an agreement between histologic and clinical response in both group I $(p=1.000)$ and group II $(p=0.344)$ but not in group III $(p=0.006)$ (Table 2$)$. 


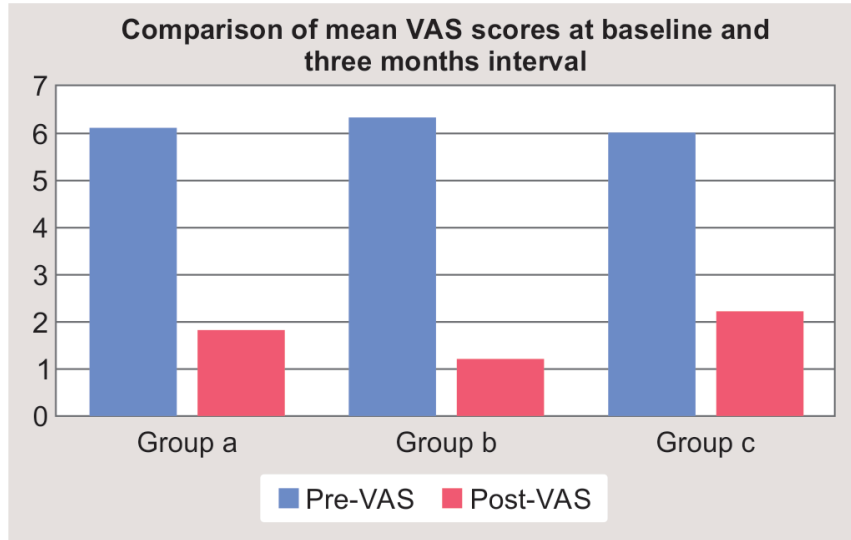

Fig. 1: Intergroup comparison of mean VAS scores at baseline and 3 months' interval
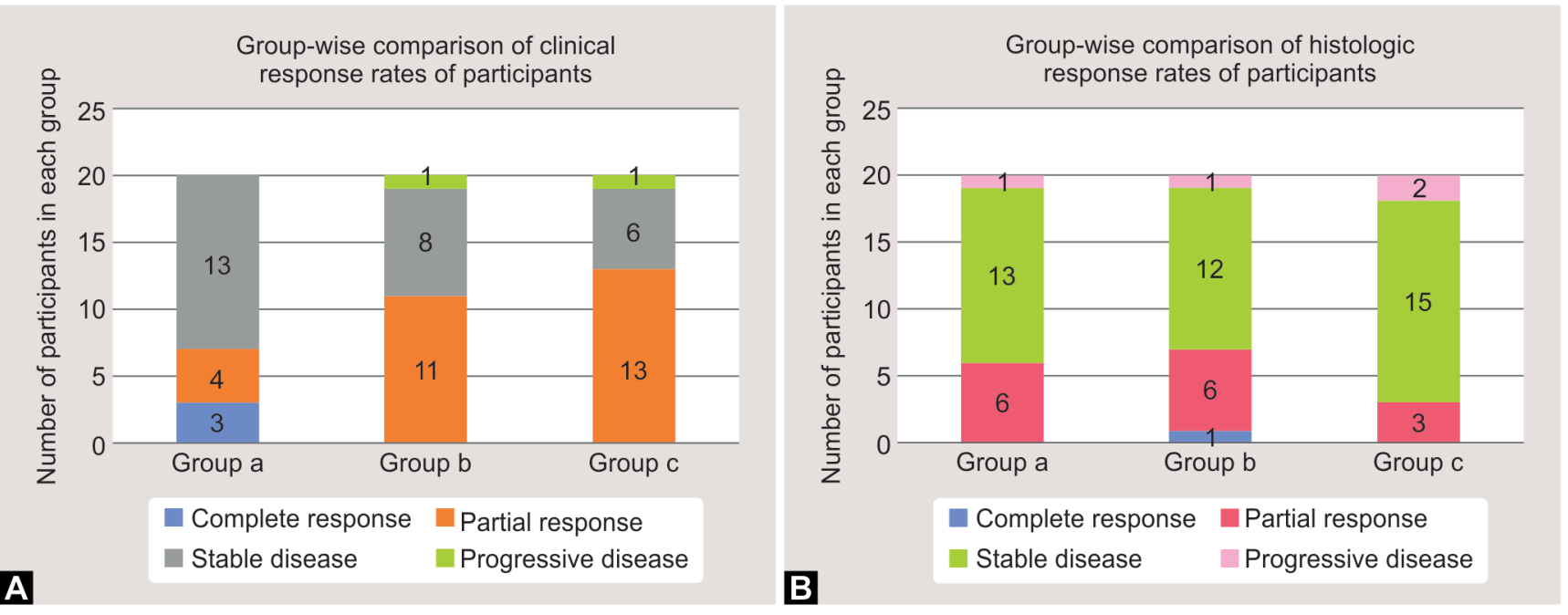

Figs 2A and B: (A) Group-wise comparison of clinical response rates of participants; (B) Group-wise comparison of histological response rates of participants

Among all the patients who completed the follow-up, six patients ultimately developed oral malignancy and 25 months was the median follow-up time. 31.5 months was the median time for oral cancer development. Between the three treatment arms, there was no statistically significant difference $(p>0.05)$ in oral cancer-free existence. None of the clinical or histologic responders to the study medications were associated with oral cancer development.

\section{Biomarker Results}

Baseline (B) biomarker examinations were carried out in 59 tissue specimens for Ki-67, 52 tissue specimens for cyclin D1, and 55 tissue specimens for p53 expression. The 3-month posttreatment analyzes were conducted in 56 tissue specimens for Ki-67, 53 tissue specimens for cyclin D1, and 59 tissue specimens for $\mathrm{p} 53$ expression. Some of the tissues were lost during antigen retrieval and due to folding of sections. The number of tissue specimens analyzed after the review is represented in the form of a flowchart (Flowchart 2).

In the green tea treatment arm, treatment with green tea led to lower levels of expression of cyclin D1 at 3 months. There was a nonsignificant difference seen in the expression of Ki 67 and p53 between the time intervals $(p>0.05)$ but there is a trend toward the lower level of expression of both Ki67 and p53 after 3 months of treatment with green tea. In the curcumin treatment arm, treatment with curcumin led to significant lower levels of expression of Ki67 and cyclin D1 at 3 months $(p<0.01$, 0.05 ). However, there was a statistically nonsignificant difference seen for the values between the time intervals $(p>0.05)$ for $p 53$. In the combination arm, treatment with both topical and systemic curcumin and green tea led to a significant decrease in the level of expression of all the three biomarkers, namely, Ki67, cyclin D1, and $\mathrm{p} 53$ at 3 months ( $p=0.022,0.011,0.031$, respectively). Figure 3 represents biomarker modulation (labeling index values) in all the three study groups. Figure 4 illustrates examples of biomarker modulation to combination treatment at baseline and 3 months in IHC-stained sections.

When intensity of staining was evaluated at baseline in the curcumin arm and the green tea arm, it was found that there was a higher frequency of moderate basal and suprabasal staining for cyclin D1 and P53 and after 3 months of treatment, which led to higher frequency of mild basal staining of cyclin D1 $(p<0.01)$ and P53 $(p>0.05)$. In the combination arm, though there was decrease in number of cases with moderate basal and suprabasal staining after 3 months, the intensity grading between the time intervals 
Flowchart 2: Flow diagram showing tissues available for biomarker analysis

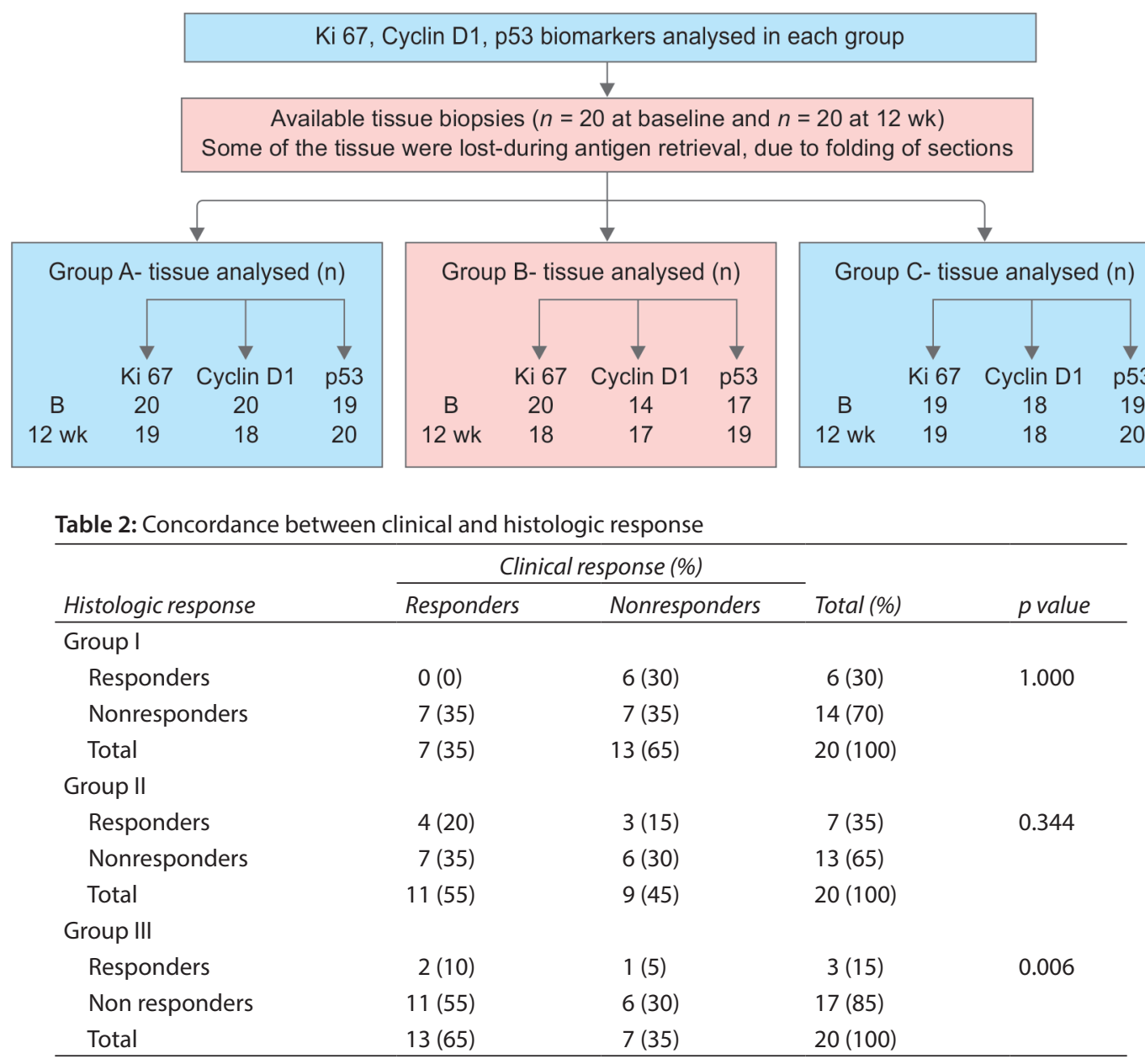

(i.e., B vs. 3 months) for both cyclin D1 and p53 was not statistically significant $(p>0.05)$.

There was increase in baseline mean values of Ki67, p53, and cyclin D1 labeling index with increasing degree of dysplasia. When baseline biomarker levels were correlated with the risk profile, there was a significant difference in the levels of all the three biomarkers among patients with habits and without habits (Table 3). Mean p53, cyclin D1, and Ki67 levels among tobacco chewers, smokers, and drinkers were significantly higher when compared to patients without any habits ( $p<0.05$ except for cyclin D1 in smokers were $p$ value was 0.102 , Wilcoxon signed-rank test).

\section{Safety Results}

The treatment was safe with intervention-related adverse effects informed by 31 of the 60 (51.6\%) patients who were on treatment medications. Table 4 reviews the most common adverse effects among the three groups. The most often grade I-II adverse events (AEs) described were abdominal pain, nausea, insomnia, diarrhea, headache, constipation, flatulence, and gastric reflux. There was no grade III, IV, or V adverse events reported. The daily dose of all the study medications was well accepted by the patients in all the three treatment groups. There is no statistically significant difference in AEs between the green tea arm, curcumin, and combination arms $(p>0.05)$. None of the patients discontinued therapy due to side effects.

\section{Discussion}

Over the course of time, numerous efforts have been made to formulate a harmless and clinically effectual chemopreventive agent. Chemopreventive approaches for OPMDs have been hindered by the high rate of recurrence, incidence of adverse effects, and increase in mortality with the interventions such as retinoid and its analogs, $\beta$-carotene, $a$-tocopherol, and selective Cox 2 inhibitor, Celecoxib. ${ }^{2,3,18-21}$ In the present study, we found the combination of green tea and curcumin to be a novel and safe modality to treat OPMDs as it resulted in a tendency toward a greater clinical response in association with biomarker modulation that was ascertained by the high baseline level of p53, cyclin D1, and Ki 67 and downregulation of all these biomarkers after 3 months of therapy. Thus, the effectiveness of green tea and curcumin combination has been validated with the use of suitable biomarkers.

In the current research, we used natural dietetics because they are composed of a varied range of biologically potent phytochemicals like phenols, bioflavonoids, tetraterpenes, alkaloids, and nitrogen that have the ability to restrain early and late phases of carcinogenesis. ${ }^{22}$

Green tea being superabundant with polyphenols has shown to hinder a range of actions related to tumor cell development, persistence, and also metastasis. Green tea when combined with curcumin, another popular natural compound, has shown 


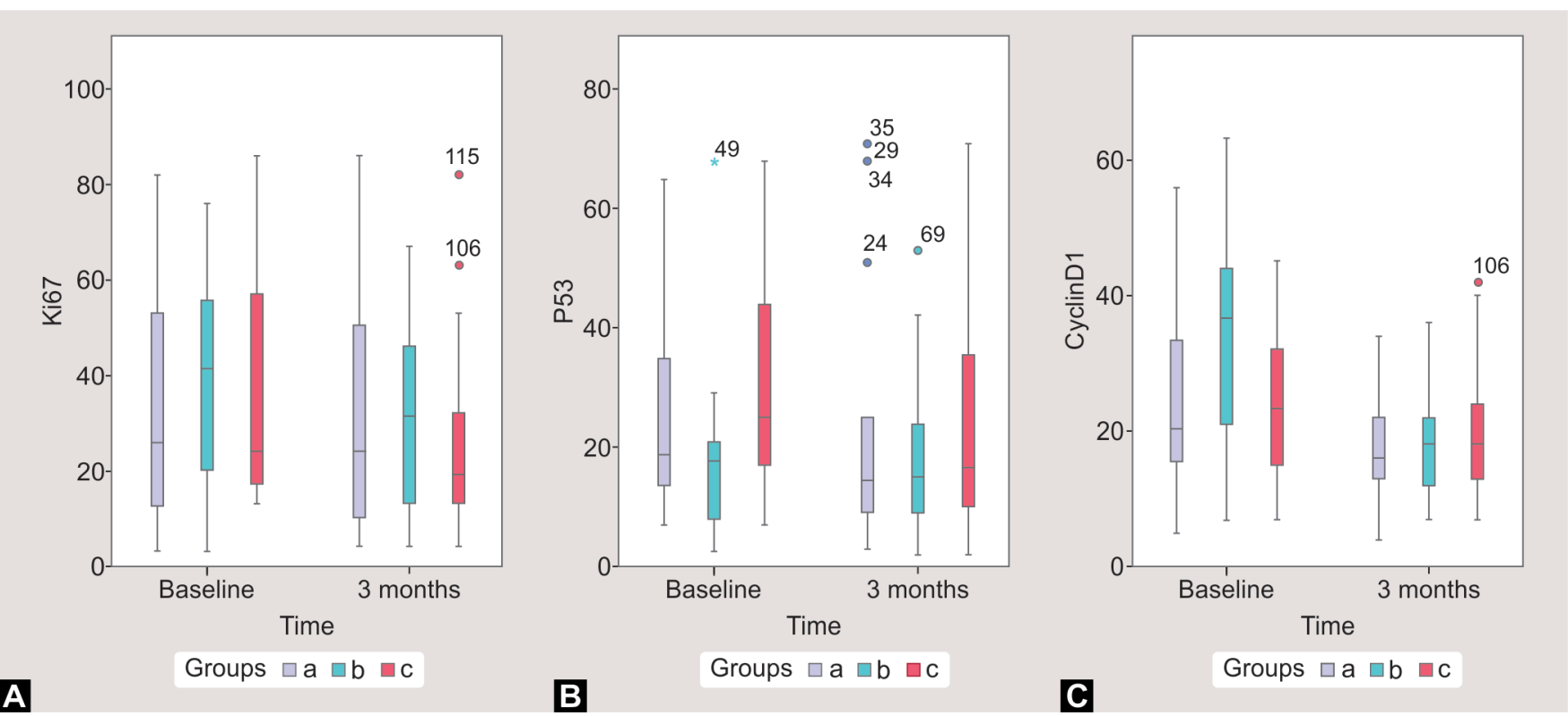

Figs 3A to C: Box plot showing baseline and 3 months' interval Ki 67 (A), p53 (B), cyclin D1 (C) labeling index (LI) data related to three treatment groups (group I-green tea arm, group II—curcumin arm, group III—combination arm). Bars within each box indicate median LI values; bottom and top of each box denotes 25 th and 75 th percentile values, respectively
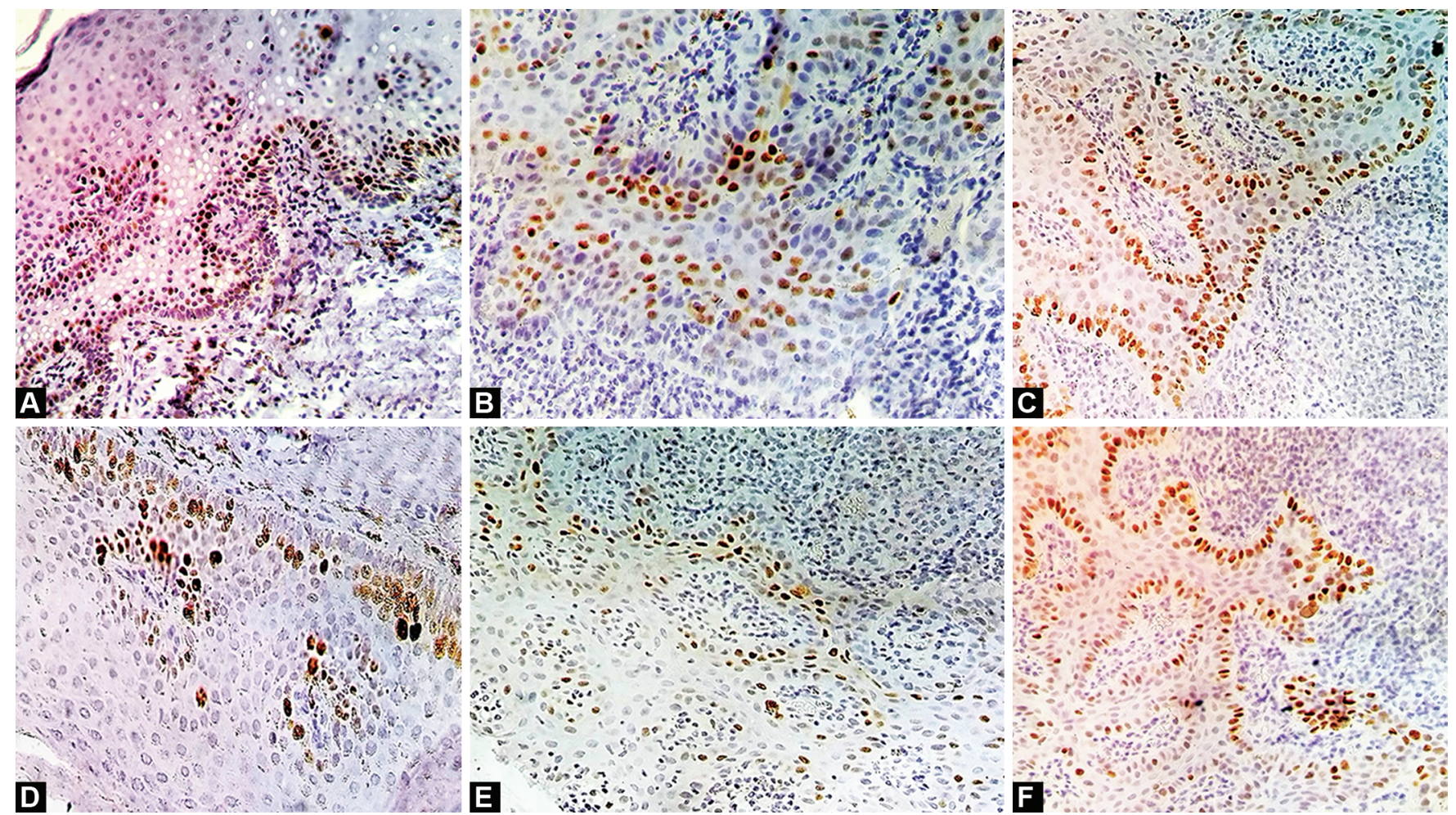

Figs 4A to F: (A) Immunohistochemical staining of Ki 67 before treatment ( $\times 10$ magnification); (B) same patient showing downregulation of Ki 67 after treatment ( $\times 40$ magnification); (C) immunohistochemical staining of cyclin D1 before treatment $(\times 40$ magnification); (D) same patient showing downregulation of cyclin D1 after treatment ( $\times 10$ magnification); $(E)$ immunohistochemical staining of p53 before treatment $(\times 10$ magnification); (F) same patient showing downregulation of p53 after treatment ( $\times 10$ magnification)

synergistic growth-constraining properties in head and neck cancer as well as other cancer cell lines. ${ }^{11-13}$ So we decided to use green tea with curcumin to establish the synergistic effect in vivo to treat patients with OPMDs. To our knowledge, it is the first study to use combination of green tea and curcumin in vivo among patients with OPMDs and at the same time assess pre- and posttreatment biomarker levels.

Natural chemopreventive agents are known to control important molecular targets like EGFR, caspase, VEGF-1, p14/16, p53, p21, p27, p57, Ki 67, cyclin D1, and cyclin E. ${ }^{23}$ In our study 
Table 3: Baseline biomarker with risk profile of patients

\begin{tabular}{|c|c|c|c|c|c|c|c|}
\hline Biomarkers & Habits & $n$ & Mean & $S D$ & Mean rank & $Z$ & $p$ \\
\hline & Smoking status & & & & & & \\
\hline \multirow[t]{2}{*}{ P53 } & Nonsmokers & 32 & 25.0926 & 17.59531 & 24.09 & -1.922 & 0.055 \\
\hline & Smokers & 22 & & & 32.45 & & \\
\hline \multirow[t]{2}{*}{ Cyclin D1 } & Nonsmokers & 33 & 27.5192 & 14.46902 & 23.89 & -1.636 & 0.102 \\
\hline & Smokers & 19 & & & 31.03 & & \\
\hline \multirow[t]{3}{*}{ Ki67 } & Nonsmokers & 34 & 35.6842 & 23.41715 & 24.15 & -2.686 & 0.007 \\
\hline & Smokers & 23 & & & 36.17 & & \\
\hline & Smokeless status & & & & & & \\
\hline \multirow[t]{2}{*}{ P53 } & Nonchewers & 18 & 25.0926 & 17.59531 & 17.86 & -3.189 & 0.001 \\
\hline & Chewers & 36 & & & 32.32 & & \\
\hline \multirow[t]{2}{*}{ Cyclin D1 } & Nonchewers & 20 & 27.5192 & 14.46902 & 17.55 & -3.369 & 0.001 \\
\hline & Chewers & 32 & & & 32.09 & & \\
\hline \multirow[t]{3}{*}{ Ki67 } & Nonchewers & 20 & 35.6842 & 23.41715 & 16.88 & -4.058 & 0.000 \\
\hline & Chewers & 37 & & & 35.55 & & \\
\hline & Alcohol status & & & & & & \\
\hline \multirow[t]{2}{*}{ P53 } & Nonalcoholics & 47 & 25.0926 & 17.59531 & 25.69 & -2.192 & 0.028 \\
\hline & Alcoholics & 7 & & & 39.64 & & \\
\hline \multirow[t]{2}{*}{ Cyclin D1 } & Nonalcoholics & 46 & 27.5192 & 14.46902 & 24.41 & -2.752 & 0.006 \\
\hline & Alcoholics & 6 & & & 42.50 & & \\
\hline \multirow[t]{2}{*}{ Ki67 } & Non alcoholics & 51 & 35.6842 & 23.41715 & 27.46 & -2.043 & 0.041 \\
\hline & Alcoholics & 6 & & & 42.08 & & \\
\hline
\end{tabular}

Table 4: Common adverse effects among the groups

\begin{tabular}{lllll}
\hline & \multicolumn{4}{c}{ Group } \\
\cline { 2 - 4 } Adverse effects & $I(\%)$ & $I I(\%)$ & III (\%) & Total (\%) \\
\hline Abdominal pain & $0(0)$ & $4(20)$ & $5(25)$ & $9(15)$ \\
Constipation & $1(5)$ & $1(5)$ & $0(0)$ & $2(3.3)$ \\
Diarrhea & $2(10)$ & $1(5)$ & $1(5)$ & $4(6.6)$ \\
Flatulence, gastric reflux & $1(5)$ & $1(5)$ & $0(0)$ & $2(3.3)$ \\
Gastric reflux & $1(5)$ & $1(5)$ & $0(0)$ & $2(3.3)$ \\
Headache & $0(0)$ & $1(5)$ & $2(10)$ & $3(5)$ \\
Nausea & $1(5)$ & $2(10)$ & $2(10)$ & $5(8.3)$ \\
Nausea, abdominal pain & $0(0)$ & $1(5)$ & $0(0)$ & $1(1.6)$ \\
Insomnia & $2(10)$ & $0(0)$ & $1(5)$ & $3(5)$ \\
No side effects & $12(60)$ & $8(40)$ & $9(45)$ & $29(48.3)$ \\
Total & 20 & 20 & 20 & 60 \\
\hline
\end{tabular}

we chose to use cell growth/proliferation markers-Ki67, cyclin D1, and apoptosis marker-p53, as they can aid in defining the biological action of any chemopreventive agent successfully in a clinical setting.

In most of the chemoprevention trials, incidence of cancer has been used as the primary endpoint, but we have employed clinical response and biomarker assessment as surrogate primary endpoints because it becomes essential to have trials with long follow-up time, large sample size, and substantial financial resources if cancer incidence is considered as the endpoint. ${ }^{24}$

In the present study, clinical response $(C R+P R)$ was observed in $65 \%$ of subjects in the combination arm, $55 \%$ in the curcumin arm, and $35 \%$ subjects in the green tea arm. The combination (curcumin + green tea) arm showed significant difference in the clinical response rate as compared to the green tea and curcumin arm. These results establish the possible synergistic action of green tea and curcumin. The histologic response as an endpoint has yielded unreliable outcomes, so it has been uncommonly used in oral chemoprevention studies. ${ }^{25}$ Individual judgment of dysplastic changes, diagnostic standards employed, and enormous interobserver variance are identified as the possible reasons. In the current study, we were not able to recognize significant improvement in the histologic response; even though there was a better clinical response, histological response (CR + PR) was observed in $15 \%$ of subjects in the combination arm, 35\% in the curcumin arm, and $30 \%$ in the green tea arm. The possible explanation for it could be that biopsies were obtained from the remaining lesion tissue, not from the nearby responding tissue, in which modulation may have ensued.

The phase 1 trial using curcumin in high-risk or premalignant lesions found histologic improvement in $35 \%$ of patients, which was similar to our study. ${ }^{26}$ Phase $2 \mathrm{~B}$ trial using curcumin in patients with 
leukoplakia showed a significant clinical response but the histologic response was not significant. ${ }^{27}$ Other studies using systemic curcumin showed a significant clinical improvement in leukoplakia and OSMF patients. ${ }^{28,29}$ Curcumin when used as a sole systemic treatment for oral lichen planus, significant clinical improvement has been observed whereas another study concluded curcumin to be ineffective in the treatment of oral lichen planus. ${ }^{30,31}$

The phase 2 trial using green tea in high-risk oral premalignant lesions observed similar clinical response of 36.4 and $27.2 \%$ of the histologic response at the dose of green tea used in our study and they also noticed a better clinical response but no histologic response and higher incidence of adverse effects at greater doses of green tea. ${ }^{1}$ The phase 1 trial conducted to investigate the antitumor response of oral green tea extract on solid tumors including head and neck cancers found no major or minor antitumor effects. ${ }^{32}$ Clinical and histologic improvement following topical application of green tea extract has also been observed in a study on oral leukoplakia. ${ }^{33}$ In an in vitro multistage carcinogenesis model, green tea extract was found to inhibit leukoplakia cell lines effectively. ${ }^{4}$

There are no human studies using combination of green tea and curcumin in any oral lesions but combination of green tea and curcumin on animal models has resulted in greater preventive activity than using these agents singly. Epigallacatechin-3-gallate promotes keratinocyte differentiation, and EGCG and curcumin together suppress keratinocyte proliferation and survival. Green tea catechins may further enhance the uptake of curcumin and overcome its low bioavailability. ${ }^{11-13}$

There was significant symptomatic improvement in all the three treatment groups in our study. The VAS with higher values in all the treatment groups reduced significantly following treatment with study medications after 12 weeks. This may be due to combination of topical and systemic medications in all the treatment arms. Topical preparations not only deliver high concentration of the medicament to act locally on the lesion tissue but also will help to reduce the dose of systemic preparations and this has been one of the prime reasons for using a lower dose of study medications in the treatment groups. Symptomatic relief obtained in our study is similar to other studies using curcumin and green tea singly for the treatment of lesions like leukoplakia, oral lichen planus, and OSMF. ${ }^{33-35}$

Biomarker assessments and interpretation of tissues in clinical studies are laborious and very demanding. Ki-67, p53, and Cyclin D1 are the three tissue-based biomarkers assessed. The difficulties ranged from tissue loss during antigen retrieval, folding of sections, and thick sections $(4 \mu \mathrm{M})$. Combination of green tea and curcumin in this study produced a good clinical response together with a significant downregulation of all the biomarkers assessed p53, cyclin D1, and Ki 67 after 3 months (posttreatment). Numerous studies have been reported in the literature investigating the role of green tea supplements on molecular markers of cancers. It has been proposed that EGCG-mediated growth arrest and apoptosis can occur via p53-dependent and independent pathways. ${ }^{36}$ Epigallacatechin-3-gallate downregulated cyclin D1 and p21 transcriptional activation partly promoted growth arrest in several different cancer cells. ${ }^{37}$ Green tea supplementation resulted in reduction of Ki- 67 levels in both benign and malignant cell lines. ${ }^{38}$ Curcumin treatment has been found to be efficacious in cancer cells that demonstrate high magnitude of mutant p53 than those that manifest a wild-type p53. ${ }^{8}$ Curcumin downgraded cyclin D1 levels and restrained Cdk4-mediated phosphorylation of retinoblastoma protein, leading to growth arrest of tumor cells. ${ }^{9}$ Curcumin reduced expression of Ki67 both in vitro and in vivo, thus exhibiting a strong antiproliferative effect. ${ }^{10}$ P53 is mutated in more than $50 \%$ of cancers; carcinomas of oral cavity often contain mutated p53 and higher expression of it has been found in OPMDS, so the p53 downregulation observed in our study is that of a mutant type of p53 not the wild-type p53. ${ }^{14,39}$

Downregulation of biomarker levels observed in the present study has been further strengthened by a decrease in the intensity of cyclin D1 and p53 staining between the time periods in all the three groups. We also observed an increase in the Ki67, p53, and cyclin D1 labeling index with increasing degree of dysplasia, although the differences between the grades of dysplasia were not statistically significant. Our findings are similar to other reported studies in the literature. ${ }^{40,41}$

There was a very good agreement between clinical and histologic responders in both green tea and curcumin groups but we found that in the combination group there was no concordance between clinical and histologic responders may be due to greater number of cases with high-risk OPMDs at baseline. These cases failed to exhibit a histologic response in spite of showing a good clinical response.

The observed safety of curcumin and green tea extracts is in accordance to the earlier reports of clinical studies on curcumin and green tea extracts. Low dose of treatment agents has been found to be effective in OPMDs and combination treatment has not only increased effectiveness but has also lowered the incidence of adverse events, which may occur at higher doses of study medications used in other studies. 1,26,27,32

When risk profile and biomarker levels were analyzed, significant association was found between baseline biomarker levels and habits in the patient. These findings further emphasize the importance of habit cessation, which may have an effect on clinical and histologic outcomes. Association found further strengthens the need for cessation of habits during the study; if not, it would be a significant confounding factor for the biomarker assessment. ${ }^{27,40}$ However, some of the previous studies have also hypothesized that curcumin and green tea may have enhanced chemopreventive potential than placebo in tobacco and alcohol users. $^{42,43}$

Findings from this study suggest that combination of green tea and curcumin may serve as an effectual treatment for potentially malignant oral disorders, with good safety, clinical response, and an associated biomarker modulation but was ineffective in producing amelioration in oral cancer-free existence. Future long-term follow-up studies using large sample size and which are placebo-controlled are needed to further emphasize the safety and efficiency of these easily obtainable, natural, and noninvasive agents. Positive results of our study suggest that these chemopreventive agents could possibly stop development of cancer or a second primary tumor, which can be ascertained if future chemoprevention trials focus on incidence of cancer as the primary end point. It is also necessary to carry out other available molecular marker-based studies for risk assortment, response assessment, and to authenticate effectiveness of a chemopreventive agent.

\section{ACKNoWledgment}

The author expresses gratitude to the Department of Oral and Maxillofacial Surgery and Oral Pathology, College of Dental Sciences, Davangere, for the help rendered. Special thanks to 
Department of General Pathology, SSIMS and RC, for providing the facilities for immunohistochemical analysis.

\section{References}

1. Tsao AS, Liu D, Martin J, et al. Phase II randomized, placebo-controlled trial of green tea extract in patients with high-risk oral premalignant lesions. Cancer Prev Res 2009;2(11):931-941. DOI: 10.1158/1940-6207. CAPR-09-0121.

2. Papadimitrakopoulou VA, Lee JJ, William WN Jr, et al. Randomized trial of 13-cis retinoic acid compared with retinyl palmitate with or without beta-carotene in oral premalignancy. J Clin Oncol 2009;27(4):599-604. DOI: 10.1200/JCO.2008.17.1850.

3. Khuri FR, Lee JJ, Lippman SM, et al. Randomized phase III trial of low-dose isotretinoin for prevention of second primary tumors in stage I and II head and neck cancer patients. J Natl Cancer Inst 2006;98(7):441-450. DOI: 10.1093/jnci/djj091.

4. Khafif A, Schantz SP, Al-Rawi M, et al. Green tea regulates cell cycle progression in oral leukoplakia. Head Neck 1998;20(6):528-534. DOI: 10.1002/(sici)1097-0347(199809)20:63.0.co;2-3.

5. Sa G, Das T. Anti cancer effects of curcumin: cycle of life and death. Cell Div 2008;3(1):14. DOI: 10.1186/1747-1028-3-14.

6. Ahmad N, Cheng P, Mukhtar H. Cell cycle dysregulation by green tea polyphenol epigallocatechin-3-gallate. Biochem Biophys Res Commun 2000;275(2):328-334. DOI: 10.1006/bbrc.2000.3297.

7. Shin DM. Oral cancer prevention advances with a translational trial of green tea. Cancer Prev Res (Phila) 2009;2(11):919-921. DOI: 10.1158/1940-6207.CAPR-09-0207.

8. Talib WH, Al-Hadid SA, Ali MBW, et al. Role of curcumin in regulating p53 in breast cancer: an overview of the mechanism of action. Breast Cancer (Dove Med Press) 2018;10:207-217. DOI: 10.2147/BCTT. S167812.

9. Choudhuri T, Pal S, Das T, et al. Curcumin selectively induces apoptosis in deregulated cyclin D1-expressed cells at G2 phase of cell cycle in a p53-dependent manner. J Biol Chem 2005;280(20):20059-20068. DOI: 10.1074/jbc.M410670200.

10. Pichu S, Krishnamoorthy S, Shishkov A, et al. Knockdown of ki- 67 by dicer-substrate small interfering RNA sensitizes bladder cancer cells to curcumin-induced tumor inhibition. PLoS ONE 2012;7(11):e48567. DOI: 10.1371 /journal.pone.0048567.

11. Khafif A, Schantz SP, Chou TC, et al. Quantitation of chemopreventive synergism between (-)-epigallocatechin-3-gallate and curcumin in normal, premalignant and malignant human oral epithelial cells. Carcinogenesis 1998;19(3):419-424. DOI: 10.1093/carcin/19.3.419.

12. Eckert RL, Crish JF, Efimova T, et al. Opposing action of curcumin and green tea polyphenol in human keratinocytes. Mol Nutr Food Res 2006;50(2):123-129. DOI: 10.1002/mnfr.200500125.

13. Wang $S$, Chen $R$, Zhong Z, et al. Epigallocatechin-3-gallate potentiates the effect of curcumin in inducing growth inhibition and apoptosis of resistant breast cancer cells. Am J Chin Med 2014;42(5):1279-1300. DOI: 10.1142/S0192415X14500803.

14. Lippman SM, Shin DM, Lee JJ, et al. p53 and retinoid chemoprevention of oral carcinogenesis. Cancer Res 1995;55(1):16-19.

15. Pocock SJ, Simon R. Sequential treatment assignment with balancing for prognostic factors in the controlled clinical trial. Biometrics 1975;31(1):103-115. DOI: 10.2307/2529712.

16. Rai B, Kaur J, Jacobs R, et al. Possible action mechanism for curcumin in pre-cancerous lesions based on serum and salivary markers of oxidative stress. J Oral Sci 2010;52(2):251-256. DOI: 10.2334/ josnusd.52.251.

17. US Department of Health and Human Services, National Institute of Health, National Cancer Institute. Common Terminology Criteria for Adverse Events (CTCAE). Version 4.0. 2010. Available from: https://evs.nci.nih.gov/ftp1/ CTCAE/CTCAE_4.03_2010-0614_QuickReference_5x7.pdf.

18. Bjelakovic G, Nikolova D, Gluud LL, et al. Mortality in randomized trials of antioxidant supplements for primary and secondary prevention: systematic review and meta-analysis. JAMA 2007;297(8):842-857. DOI: 10.1001/jama.297.8.842.

19. Nagao T, Warnakulasuriya $S$, Nakamura $T$, et al. Treatment of oral leukoplakia with a low-dose of beta-carotene and vitamin C supplements: a randomized controlled trial. Int J Cancer 2015;136(7):1708-1717. DOI: 10.1002/ijc.29156.

20. Bairati I, Meyer F, Gélinas M, et al. A randomized trial of antioxidant vitamins to prevent second primary cancers in head and neck cancer patients. J Natl Cancer Inst 2005;97(7):481-488. DOI: 10.1093/jnci/ dji095.

21. Papadimitrakopoulou VA, William WN, Dannenberg AJ, et al. Pilot randomized phase ll study of celecoxib in oral premalignant lesions. Clin Cancer Res 2008;14(7):2095-2101. DOI: 10.1158/1078-0432.CCR07-4024.

22. Crooker $K$, Aliani R, Ananth $M$, et al. A review of promising natural chemopreventive agents for head and neck cancer. Cancer Prev Res (Phila) 2018;11(8):441-450. DOI: 10.1158/1940-6207.CAPR17-0419.

23. Ramos S. Cancer chemoprevention and chemotherapy: dietary polyphenols and signalling pathways. Mol Nutr Food Res 2008;52(5):507-526. DOI: 10.1002/mnfr.200700326.

24. Wirth LJ, Krane JF, Li Y, et al. A pilot surrogate endpoint biomarker study of celecoxib in oral premalignant lesions. Cancer Prev Res (Phila) 2008;1(5):339-348. DOI: 10.1158/1940-6207.CAPR07-0003.

25. Mehanna HM, Rattay T, Smith J, et al. Treatment and follow-up of oral dysplasia-a systematic review and meta-analysis. Head Neck 2009;31(12):1600-1609. DOI: 10.1002/hed.21131.

26. Cheng AL, Hsu CH, Lin JK, et al. Phase I clinical trial of curcumin, a chemopreventive agent, in patients with high-risk or pre-malignant lesions. Anticancer Res 2001;21(4B):2895-2900.

27. Kuriakose MA, Ramdas K, Dey B, et al. A randomized double-blind placebo-controlled phase IIB trial of curcumin in oral leukoplakia. Cancer Prev Res (Phila) 2016;9(8):683-691. DOI: 10.1158/1940-6207. CAPR-15-0390.

28. Bhagat V, Arora P, Ranjan V, et al. Efficacy of curcumin in the treatment of oral leukoplakia-a prospective study. Int J Curr Med Pharmaceut Res 2018;04(12):3959-3962.

29. Piyush P, Mahajan A, Singh K, et al. Comparison of therapeutic response of lycopene and curcumin in oral submucous fibrosis: $A$ randomized controlled trial. Oral Dis 2019;25(1):73-79. DOI: 10.1111/ odi.12947.

30. Kia SJ, Basirat M, Estakhr L. The effect of oral curcumin on pain and clinical appearance of oral lichen planus. J Dentomaxillofac Radiol Pathol Surg 2017;6(1):1-7.

31. Amirchaghmaghi M, Pakfetrat A, Delavarian Z, et al. Evaluation of the efficacy of curcumin in the treatment of oral lichen planus: a randomized controlled trial. J Clin Diagn Res 2016;10(5):ZC134-ZC137. DOI: 10.7860/JCDR/2016/16338.7870.

32. Pisters KM, Newman RA, Coldman B, et al. Phase I trial of oral green tea extract in adult patients with solid tumors. J Clin Oncol 2001;19(6):1830-1838. DOI: 10.1200/JCO.2001.19.6.1830.

33. Katakura A, Shibahara T, Inoue K, et al. Therapeutic effect of green tea catechin on oral leukoplakia. Cancer Epidemiol Biomarkers Prev 2006;15(12):B148.

34. Kia SJ, Shirazian S, Mansourian A, et al. Comparative efficacy of topical curcumin and triamcinolone for oral lichen planus: a randomized, controlled clinical trial. J Dent (Tehran) 2015;12(11):789-796.

35. Lanjekar AB, Bhowate RR, Bakhle $S$, et al. Comparison of efficacy of topical curcumin gel with triamcinolone hyaluronidase gel individually and in combination in the treatment of oral submucous fibrosis. J Contemp Dent Pract 2020;21(1):83-90. DOI: 10.5005/ jp-journals-10024-2726.

36. Gupta K, Thakur VS, Bhaskaran N, et al. Green tea polyphenols induce p53-dependent and p53-independent apoptosis in prostate cancer cells through two distinct mechanisms. PLoS ONE 2012;7(12):e52572. DOI: 10.1371/journal.pone.0052572. 
37. Zhang X, Min KW, Wimalasena J, et al. Cyclin D1 degradation and p21 induction contribute to growth inhibition of colorectal cancer cells induced by epigallocatechin-3-gallate. J Cancer Res Clin Oncol 2012;138(12):2051-2060. DOI: 10.1007/s00432-0121276-1.

38. Yu SS, Spicer DV, Hawes D, et al. Biological effects of green tea capsule supplementation in pre-surgery postmenopausal breast cancer patients. Front Oncol 2013;3:298. DOI: 10.3389/fonc.2013. 00298.

39. Sakai E, Tsuchida N. Most human squamous cell carcinomas in the oral cavity contain mutated p53 tumor-suppressor genes. Oncogene 1992;7(5):927-933.

40. Ramakrishna A, Shreedhar B, Narayan T, et al. Cyclin D1 an early biomarker in oral carcinogenesis. J Oral Maxillofac Pathol 2013;17(3):351-357. DOI: 10.4103/0973-029X.125189.

41. Raju B, Mehrotra R, Øijordsbakken G, et al. Expression of p53, cyclin $\mathrm{D} 1$ and $\mathrm{Ki}-67$ in pre-malignant and malignant oral lesions: association with clinicopathological parameters. Anticancer Res 2005;25(6C):4699-4706.
42. Klaunig JE, Xu Y, Han $\mathrm{C}$, et al. The effect of tea consumption on oxidative stress in smokers and nonsmokers. Proc Soc Exp Biol Med 1999;220(4):249-254. DOI: 10.3181/00379727-220-44375.

43. Shishodia S, Potdar P, Gairola CG, et al. Curcumin (diferuloylmethane) down-regulates cigarette smoke-induced NF-kB activation through inhibition of IKBa kinase in human lung epithelial cells: correlation with suppression of COX-2, MMP-9 and cyclin D1. Carcinogenesis 2003;24(7):1269-1279. DOI: 10.1093/carcin/bgg078.

44. Bouquot JE, Whitaker SB. Oral leukoplakia-rationale for diagnosis and prognosis of its clinical subtypes or" phases". Quintessence Int 1994;25(2):133-140.

45. Thongprasom K, Luangjarmekorn L, Sererat T, et al. Relative efficacy of fluocinolone acetonide compared with triamcinolone acetonide in treatment of oral lichen planus. J Oral Pathol Med 1992;21(10):456458. DOI: 10.1111/j.1600-0714.1992.tb00974.x.

46. Kerr AR, Warnakulasuriya S, Mighell AJ, et al. A systematic review of medical interventions for oral submucous fibrosis and future research opportunities. Oral Dis 2011;17(Suppl 1):42-57. DOI: 10.1111/j.16010825.2011.01791.x. 